\title{
Interplay of quantum and thermal fluctuations in a frustrated magnet
}

\author{
S. V. Isakov ${ }^{1}$ and R. Moessner ${ }^{2}$ \\ ${ }^{1}$ Department of Physics, Stockholm University, AlbaNova, S-106 91 Stockholm, Sweden and \\ ${ }^{2}$ Laboratoire de Physique Théorique de l'Ecole Normale Supérieure, CNRS-UMR8549, Paris, France
}

\begin{abstract}
We demonstrate the presence of an extended critical phase in the transverse field Ising magnet on the triangular lattice, in a regime where both thermal and quantum fluctuations are important. We map out a complete phase diagram by means of quantum Monte Carlo simulations, and find that the critical phase is the result of thermal fluctuations destabilising an order established by the quantum fluctuations. It is separated by two Kosterlitz-Thouless transitions from the paramagnet on one hand and the quantum-fluctuation driven three-sublattice ordered phase on the other. Our work provides further evidence that the zero temperature quantum phase transition is in the $3 \mathrm{~d} X Y$ universality class.
\end{abstract}

\section{INTRODUCTION}

In geometrically frustrated magnets, the arrangement of the spins on a lattice precludes the establishment of a simple collinear Néel state. In the absence of such an 'obvious' ordering pattern, these magnets are often subject to a large number of competing instabilities. This endows them with a rich behaviour, manifested in unconventional phases, phase transitions and excitations, a large low-energy density of states and unusual dynamical properties. 1

When added to a frustrated magnet which is not ordered classically at temperature $T=0$, both thermal and quantum fluctuations often generate an ordering transition, a process known as order by disorder 2.3.4 Whereas quantum order by disorder occurs in a fashion quite analogous to the ordering induced by thermal fluctuations, the nature of the ordering (if any) of even some 'simple' model systems (such as the Heisenberg magnet on the pyrochlore lattice) in the presence of stronger quantum fluctuations remains unclear.

The interplay of thermal and quantum fluctuations has so far not received a great deal of theoretical attention for frustrated magnets. By contrast, there has been a considerable amount of interest devoted to the real-time dynamics and transport at finite temperature near quantum critical points ${ }^{5}$ In addition, this combination has even been claimed to provide an alternative use of quantum effects for speeding up computations. 6

In this publication, we study the combined effect of thermal and quantum fluctuations on possibly the simplest quantum frustrated magnet, namely an Ising model on the triangular lattice in a transverse magnetic field. The manifold of classical ground states is hugely degenerate, and correlations averaged over it are critical 7 This model thus enables us to investigate how the two types of fluctuations together establish and destroy order out of the exponentially large set of classical ground states. By changing the values of transverse field, $\Gamma$, and temperature, $T$, their strengths are in principle separately experimentally tunable.

We map out the phase diagram of the Ising model on the triangular lattice in the transverse field-temperature plane. The bulk of the paper is devoted to a numerical study based on a continuous time quantum Monte Carlo algorithm. We find that there are three different phases. In the regime where quantum fluctuations are weak, yet dominate over thermal fluctuations, they generate a three-sublattice ordered phase. Upon increasing the strength of thermal fluctuations, it 'melts' into a critical phase which has a finite extent and displays drifting exponents. This dome-shaped critical phase is delineated above and below by Kosterlitz-Thouless (KT) transitions at finite temperature. At $T=0$, it terminates in the classical critical point at zero field and in a quantum phase transition at a finite $\Gamma_{c}$. At high temperatures and large field strengths, one retrieves a simple paramagnet - order by disorder is a reentrant phenomenon. We briefly comment on the advantages of different diagnostics for the floating KT phase.

The results reported here largely build on the following pieces of work. In Ref. 8, a number of frustrated transverse field Ising models were studied, and a connection was pointed out between these models and classical stacked magnets in a scaling limit. An influential paper by Blankschtein and coworkers $\frac{9}{}$ on stacked triangular magnets derived a Landau-Ginzburg-Wilson theory for these systems, and predicted the stacked problem to be in the universality class of the $X Y$ model in three dimensions with a six-state clock term, a problem which has in turn been studied by Jose et al. ${ }^{10}$ Collecting these results together led to a conjecture of the phase diagram we map out in the following ${ }^{8}$. We note that, by adding a continuous degree of freedom to a classical triangular Ising antiferromagnet, a floating critical phase has previously been obtained by Chandra et al. ${ }^{11}$

The general validity of the Landau-Ginzburg approach has been somewhat called into question by Monte Carlo simulations on the ferromagnetically stacked triangular Ising antiferromagnet, with several groups presenting evidence in favour of critical behaviour in the threedimensional universality class, while others found a different, possibly new, universality class to be a more likely scenario. 12.13.14.15.16 Our work here lends further support to the Landau-Ginzburg approach which is independent of the previous numerical simulations. 


\section{MODEL}

Possibly the simplest model which has both a high classical ground state degeneracy and a non-trivial quantum dynamics is the transverse magnetic field antiferromagnetic Ising model on the triangular lattice. The model has the following Hamiltonian:

$$
H=J \sum_{\langle i, j\rangle} \sigma_{i}^{z} \sigma_{j}^{z}-\Gamma \sum_{i} \sigma_{i}^{x},
$$

where $J>0$ is nearest-neighbour exchange couplings and $\Gamma$ is the magnetic field strength.

The classical model triangular Ising magnet is obtained in the absence of a transverse field, $\Gamma=0$. Its solution is well known: ${ }^{7}$ it is disordered at any finite temperature, and critical at $T=0$, where it retains an extensive zero point entropy, $\mathcal{S}$, with $\mathcal{S} / N=0.323 k_{B}$, where $N$ is the number of spins and $k_{B}$ denotes the Boltzmann constant. Upon application of an infinitesimal transverse field, $\Gamma=$ $0^{+}$, at $T=0$, the magnet orders into a three sublattice $\sqrt{3} \times \sqrt{3}$ pattern with a sublattice magnetisation pattern $(1,0,-1) .8$

\section{A. Mapping to a stacked magnet}

Using the Suzuki-Trotter formalism, 17.18 one can map the d-dimensional quantum model onto the $(d+1)$ dimensional classical problem. The partition function of the quantum Hamiltonian (Eq. 1), Tr $\exp (-\beta H)$, is equivalent to the following partition function:

$$
\begin{gathered}
Z=\operatorname{Tr} \exp \left(-S_{c l}\right), \\
S_{c l}=K \sum_{\langle i, j\rangle, \tau} S_{i, \tau} S_{j, \tau}-K^{\tau} \sum_{i, \tau} S_{i, \tau} S_{i, \tau+1},
\end{gathered}
$$

where $S_{i, \tau}$ are classical Ising spins taking values 1 or -1 ; $\tau$ is the index in the imaginary time direction and runs from 0 to $N, N=\beta / \epsilon$ is the number of slices in the imaginary time direction and $\epsilon$ is the imaginary time step; $K=J \epsilon$ and $K^{\tau}=-\frac{1}{2} \ln \tanh \epsilon \Gamma$. The mapping becomes exact in the scaling limit $\epsilon \rightarrow 0, K^{\tau} \rightarrow \infty$, while maintaining

$$
e^{-2 K^{\tau}}=\epsilon \Gamma
$$

It is in this formulation that the dimensional crossover between the high temperature $d=2$ and the low temperature $d=3$ behaviour is most transparent. Besides providing an efficient Monte Carlo algorithm (see below), the above mapping also allows us to make contact with the literature on stacked triangular magnets, 16 from which our problem can be obtained in the scaling analysis of infinite coupling strength in the third dimension, with the third dimension being of finite length at nonzero temperature.

\section{B. LGW analysis}

Blankschtein et $a l^{9}{ }^{\frac{9}{}}$ have identified a complex XY order parameter for a stacked triangular antiferromagnet: the amplitude and phase of the Fourier mode at $\vec{Q}_{ \pm}=( \pm 4 \pi / 3,0), \psi_{ \pm}=m \exp ( \pm i \phi)$. They have obtained the following LGW Hamiltonian (see below):

$$
\begin{aligned}
H_{L G W}= & \sum_{\vec{q}}\left(r+q^{2}\right) m^{2}+u_{4} m^{4} \\
& +u_{6} m^{6}+v_{6} m^{6} \cos (6 \theta) .
\end{aligned}
$$

A six-fold clock anisotropy thus appears at sixth order in $m$. The six-fold clock term is irrelevant at $d=3$ so that the transition into a phase with nonzero $m$ should be in the $d=3 \mathrm{XY}$ universality class. This applies both to the zero temperature quantum phase transition as we vary $\Gamma$ and to the corresponding transition in a stacked Ising magnet. With the clock term being dangerously irrelevant, the transition is immediately into a state with a broken clock symmetry, the details of which depend on the sign of $v_{6}$ (see below).

At finite temperatures the triangular transverse field Ising model maps onto a stacked magnet of finite size, $L_{\tau}$, in the temporal direction, which is effectively 2dimensional as the temporal correlation length, $\xi_{\tau}$, exceeds $L_{\tau}$ close to a continuous phase transition. The 2-dimensional six-state clock model, studied by Jose et al. ${ }^{10}$ has a remarkable phase diagram consisting of three phases: a disordered phase, an extended intermediate KT phase, and an ordered phase. The extended KT phase owes is existence to the fact that in $d=2$, the clock term does not become relevant immediately below the KT transition of the XY model; instead, the coupling needs to be increased further until the clock term is sufficiently strong to assert itself.

One of the central objectives of this paper is to establish the presence of this floating KT phase as a finitetemperature induced dimensional crossover in the $2+1$ dimensional quantum Ising magnet on the triangular lattice. In our context, the presence of such a phase would be the result of an XY order parameter generated by frustration and stabilised by quantum fluctuations, together with destabilising fluctuations provided by the finite temperature.

Details of the ordered state depend on the sign of $v_{6}$. $H_{L G W}$ is minimised by $M=|M| \exp (i \Phi)$, with $\Phi=n \pi / 3$ and $\Phi=(n+1 / 2) \pi / 3$ for $v_{6}<0$ and $v_{6}>0$ respectively, with $n=1 \ldots 6$. The real space configurations of the ordered phases can be obtained by Fourier transforming these modes. In the case of $v_{6}<0$, Fourier transforming yields the following magnetisations assigned to the three sublattices of the triangular lattice: $|M|(1,-1 / 2,-1 / 2)$. We depict this phase as $(+--)$. In the case of $v_{6}>0$, we have the following sublattice magnetisations: $|M|(\sqrt{3} / 2,0,-\sqrt{3} / 2)$. We depict this phase as $(+0-)$. There are six degenerate states for both $v_{6}<0$ and $v_{6}>0$. 
It is difficult to determine the sign of $v_{6}$ - in particular, in the effective Hamiltonian, its value may drift and even change sign ${ }^{9.19}$ The partition function $Z=\exp \left(-\sum_{i j} s_{i} K_{i j} s_{j}\right)$ of the Ising model $H=$ $(1 / \beta) \sum_{i j} s_{i} K_{i j} s_{j}$ is equivalent to the following partition function (see, e.g. Ref. 20)

$$
\begin{aligned}
Z \propto \int D \psi \exp (-\mathcal{L}) & =\int D \psi \exp \left\{-\sum_{i j} \psi_{i} K_{i j} \psi_{j}\right. \\
& \left.+\sum_{i} \ln \cosh \left(\sum_{j} 2 K_{i j} \psi_{j}\right)\right\}
\end{aligned}
$$

The first term is the interaction matrix, which determines the soft modes $\psi_{ \pm}$to be located at $Q_{ \pm}$ (Eq. 17). Performing the Fourier transform and expanding $\ln \cosh (x)=\frac{x^{2}}{2}-\frac{x^{4}}{12}+\frac{x^{6}}{45}-\frac{17 x^{8}}{2520}+\ldots$, we obtain the following expression for the contribution of the non-linear term to $\mathcal{L}$

$$
\begin{aligned}
\mathcal{L}_{n l} & =-2 \sum_{\mathbf{k}} K(\mathbf{k}) K(-\mathbf{k}) \psi(\mathbf{k}) \psi(-\mathbf{k}) \\
& +\frac{4}{3} \sum_{\mathbf{k}_{i}}\left[\prod_{i=1}^{4}\left(K\left(\mathbf{k}_{i}\right) \psi\left(\mathbf{k}_{i}\right)\right) \delta^{\prime}\left(\mathbf{k}_{1}+\ldots+\mathbf{k}_{4}\right)\right] \quad(6) \\
& -\frac{64}{45} \sum_{\mathbf{k}_{i}}\left[\prod_{i=1}^{6}\left(K\left(\mathbf{k}_{i}\right) \psi\left(\mathbf{k}_{i}\right)\right) \delta^{\prime}\left(\mathbf{k}_{1}+\ldots+\mathbf{k}_{6}\right)\right] \ldots
\end{aligned}
$$

The interactions for the stacked triangular antiferromagnet are written in Fourier representation as

$$
\begin{array}{r}
K(\mathbf{k})=\sum_{k_{x}, k_{y}, k_{z}}\left(J \left[\cos \left(k_{x}\right)+\cos \left(k_{x} / 2+\sqrt{3} k_{y} / 2\right)\right.\right. \\
\left.\left.+\cos \left(k_{x} / 2-\sqrt{3} k_{y} / 2\right)\right]-J^{\prime} \cos \left(k_{z}\right)\right),
\end{array}
$$

where the sum is over the hexagonal Brillouin zone. The LGW Hamiltonian is thus constructed in terms of the coefficients $m \exp ( \pm i \phi)$ ), varying slowly in space, of the 'soft' modes $\psi_{ \pm}$.

The primed delta functions indicate that the wavevectors $\mathbf{k}_{i}$ need to add up to a reciprocal lattice vector. This requirement leads to the XY nature of the effective theory at low order. At sixth order, $\psi_{+}^{3} \psi_{-}^{3}$ and $\psi_{+}^{6}+\psi_{-}^{6}$ occur, the latter being the clock term, $m \cos (6 \theta) . v_{6}<0$ at this order. However, the $\cos (6 \theta)$ term appears at higher orders as well, e.g., $\psi_{+} \psi_{-}\left(\psi_{+}^{6}+\psi_{-}^{6}\right)$ at eighth order. Since the terms in the series have alternating sign and large coefficients, one cannot reliably determine the sign of $v_{6}$ near the transition this way.

\section{MONTE CARLO METHOD}

\section{A. The quantum Monte Carlo algorithm}

The $d=2+1$ dimensional classical problem obtained this way has only positive weights - there is no 'sign problem'. It can therefore be studied reasonably straightforwardly by Monte Carlo simulations. We thus simulate the classical problem defined by Eq. 22 However, it is difficult to simulate the discretized version of Eq. 2 because of the scaling limit (Eq. 3): in order to avoid discretisation errors, one has to take a very large ferromagnetic coupling in the imaginary time direction $K^{\tau} \rightarrow \infty$ at the same time increasing the height of the system exponentially as $e^{2 K^{\tau}}$.

In order to avoid this problem, we use a continuous time algorithm 21.22.23 The basic idea behind this algorithm is that, in the scaling limit, the density of domain walls in the imaginary time direction becomes exponentially sparse and it is thus more efficient to keep track of the location of the domain walls, using $\exp \left(2 K^{\tau}\right)$ as a unit of length. Thus, the height of the system, $N / \exp \left(2 K^{\tau}\right)=\beta /\left(\epsilon \exp \left(2 K^{\tau}\right)\right)=\beta \Gamma$, measured in units of $\exp \left(2 K^{\tau}\right)$, remains fixed in the continuum limit.

Due to the frustrated nature of our problem we cannot use a cluster algorithm ${ }^{24.25 .26}$ in the space directions as one could for the case of unfrustrated magnets; however, we can use a cluster algorithm in the time direction. The algorithm works as follows (for more details, see Ref. 23). We pick a random site on the triangular $L \times L$ lattice and build a cluster on that site in the imaginary time direction. Its length, $\tau$, is distributed according to the probability distribution $P(\tau) \propto \exp (-\Gamma \tau)-$ this prescription eliminates a 'freezing' of the algorithm due to the diverging temporal coupling. A given cluster is flipped using the Metropolis prescription in the spatial direction, i.e. we flip the cluster with probability $p=\min (1, \exp (-\Delta E))$, where $\Delta E$ is the (spatial) energy difference between the original configuration and the configuration with a flipped cluster.

One check of our Monte Carlo algorithm consisted of comparing it with the diagonalisation of a $3 \times 3$ lattice, and we have found excellent agreement between the two approaches.

\section{B. Parameters of the simulations}

We impose periodic boundary condition on the triangular lattice and performed simulations on lattices of size $L=9,12,15,18,24,30,36,48,60,75$. We have estimated the correlation time $\tau_{c}$ for different parameters. We perform usually $500 \tau_{c}$ Monte Carlo steps (MCS) for equilibration and from $10^{4} \tau_{c}$ to $5 \cdot 10^{5} \tau_{c}$ MCS for averaging.

\section{SCALING ANALYSIS}

In this section, we outline the quantities useful for detecting a possible floating KT phase and the adjacent KT phase transitions as well as the ordered phase. 


\section{A. Order parameters}

The complex XY order parameter deduced from the LGW analysis is given by:

$$
m e^{i \theta} \equiv\left(m_{1}+m_{2} e^{i \frac{4 \pi}{3}}+m_{3} e^{i \frac{-4 \pi}{3}}\right) / \sqrt{3}
$$

where the $m_{i}$ are the magnetisations of the three sublattices, and $m$ is real and positive. $m$ is close to 1 in the limit of zero temperature and vanishing transverse field. It equals zero for the disordered and $\mathrm{KT}$ phases, vanishing exponentially and algebraically in the limit the system size $L \rightarrow \infty$. There, the corresponding susceptibility is

$$
\chi=L^{2}\left\langle m^{2}\right\rangle / T
$$

To detect clock symmetry breaking, we consider

$$
c_{6}=\frac{\left\langle m^{6} \cos (6 \theta)\right\rangle}{\left\langle m^{6}\right\rangle} .
$$

It is easy to check that $c_{6}$ equals zero for disordered and $\mathrm{KT}$ phases, $c_{6}$ equals -1 for the $(+0-)$ phase, and $c_{6}$ equals 1 for the $(+--)$ phase. We have chosen not directly to average $\cos (6 \theta)$ as its value fluctuates most strongly when $m$ is small, that is to say, the ordering we are trying to determine the details of is weakest. The exponent $m^{6}$ has been chosen, somewhat arbitrarily, as the corresponding term in the LGW action is $\psi_{+}^{6}+\psi_{-}^{6}=$ $m^{6} \cos (6 \theta)$.

\section{B. Binder cumulant}

The appropriate Binder cumulant is $:^{27}$

$$
U=1-\frac{\left\langle m^{4}\right\rangle}{3\left\langle m^{2}\right\rangle^{2}} .
$$

The Binder cumulant has a scaling dimension of zero. It thus has the advantage of not requiring fitting an unknown leading exponent. In the limit $L \rightarrow \infty$, the Binder cumulant has the following behaviour: $U_{L} \rightarrow 0$ at disordered phase, $U_{L} \rightarrow 2 / 3$ at ordered phase, and $U_{L} \rightarrow U^{*}$ at a critical point. For an extended critical phase, the value of $U^{*}$ can drift, so that its value depends on the precise location within the critical phase.

Since the Binder cumulant has a scaling dimension of zero, the curves for different system sizes at a critical coupling should fall on a line of points where $U_{L}=U_{L^{\prime}}$ (at least in the region where corrections to scaling are small). As we describe below, this criterion in fact provides an overestimate of the size of the critical phase.

\section{Locating the KT transitions}

Here, we briefly describe the finite-size scaling analysis appropriate for KT transitions. This analysis follows that used by Challa and Landau. ${ }^{28}$ In order to determine the presence of a KT phase, we check if we can fit our simulations to the scaling forms predicted by KT theory 29 This has the advantage of restricting the number of fitting parameters, which in the most general case (allowing for critical exponents different from the KT ones) would be too large to be practical.

In particular, the correlation length $\xi$ and the susceptibility $\chi$ behave as

$$
\begin{aligned}
\xi \propto \exp \left(a t^{-1 / 2}\right), \\
\chi \propto \xi^{2-\eta}, \\
m \propto \xi^{-\eta / 2}
\end{aligned}
$$

where $a$ is a nonuniversal constant, $t=\left(T-T_{c}\right) / T_{c}$ is a reduced temperature, and $T_{c}$ is a critical temperature.

The finite-size scaling form of order parameter and susceptibility is given by

$$
\begin{gathered}
m_{L}=L^{-b} m_{0}(\xi / L), \\
\chi_{L}=L^{c} \chi_{0}(\xi / L),
\end{gathered}
$$

where $m_{0}$ and $\chi_{0}$ are unknown universal functions and $b$ and $c$ are constants. It follows from (12) that in the infinite system size limit, we have $b=\eta / 2$ and $c=2-\eta$. Therefore, at a critical point, one has:

$$
\begin{gathered}
m_{L} \propto L^{-\eta / 2}, \\
\chi_{L} \propto L^{2-\eta} .
\end{gathered}
$$

If we have an extended critical phase, these relations should hold over finite temperature range from the upper critical temperature $T_{2}$ to the lower critical temperature $T_{1}$. The critical exponent $\eta$ should vary continuously from $T_{2}$ to $T_{1}$. Plotting $\ln \left(m_{L}\right)$ or $\ln \left(\chi_{L}\right)$ versus $\ln (L)$, we can find the critical exponent $\eta$ at any point of the extended critical phase.

We can rewrite Eq. (13) as

$$
\begin{aligned}
& m_{L} L^{b}=m_{0}\left(L^{-1} \exp \left(a t^{-1 / 2}\right)\right), \\
& \chi_{L} L^{-c}=\chi_{0}\left(L^{-1} \exp \left(a t^{-1 / 2}\right)\right) .
\end{aligned}
$$

Eq. (16) is valid for $T<T_{1}$ and (17) is valid for $T>T_{2}$. For an appropriately chosen set of parameters $a, c, T_{1}$ the plot of $\chi_{L} L^{-c}$ versus $L^{-1} \exp \left(a t^{-1 / 2}\right)$ should collapse onto a universal curve for different system sizes $L$. The same should hold for a plot of $m_{L} L^{b}$ versus $L^{-1} \exp \left(a t^{-1 / 2}\right)$. From such a fit, the upper and lower critical temperatures can be determined.

\section{RESULTS}

In Fig. 11 we present the phase diagram that we find from Monte Carlo simulations. There are three phases: a disordered phase at high temperatures $(T \gg J)$ or large magnetic field strengths $\Gamma \gg J$, an extended KT phase at intermediate temperatures, and an $(+0-)$ ordered phase at low temperatures. 


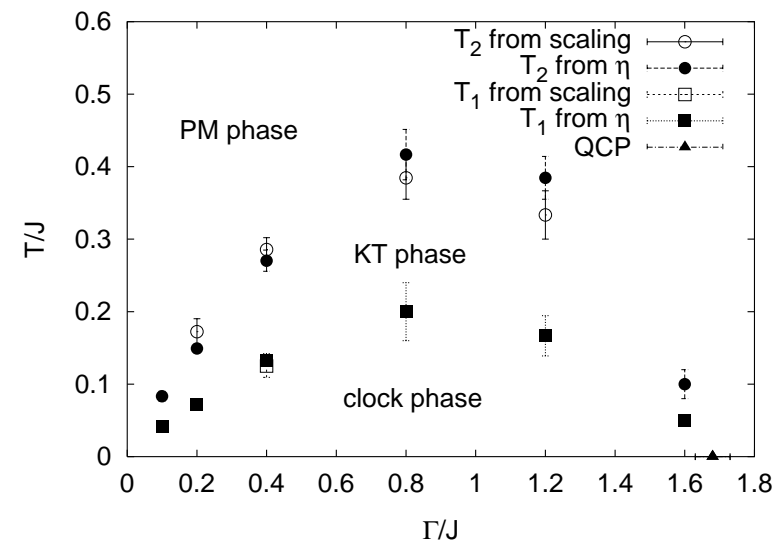

FIG. 1: The phase diagram of the transverse field Ising model on the triangular lattice. A floating KT phase separates the ordered clock phase from the paramagnet (PM). QCP denotes the location of the quantum critical point.

\section{A. The KT phase at $\Gamma / J=0.4$}

Our most complete data set was taken for $\Gamma / J=0.4$, for which we describe our analysis in detail in the next few paragraphs. We have chosen this value of $\Gamma$ as a compromise between the following requirements. Firstly, we want to stay well clear of the zero temperature critical points at $\Gamma=0$ and larger $\Gamma=\Gamma_{c}$ and concomitant possible crossover phenomena. Secondly, a high transition temperature is needed to allow us to simulate systems with only moderate extent in the imaginary time direction. Thirdly, as our algorithm slows down as the density of domain walls in the imaginary time direction increases, we would like to choose weak quantum fluctuations, that is to say, small values of $\Gamma . \Gamma / J=0.4$, located left of centre of the KT dome (Fig. 1) thus appears to be a sensible choice.

First, Fig. 2 shows the behaviour of the order parameter $m$ as a function of inverse temperature $\beta$ for different system sizes. One can notice easily that the order parameter has strong dependence on the system size in wide range of temperatures - it decreases with increasing system size.

Fig. [3 is a doubly logarithmic plot of the behaviour of the order parameter as a function of the system size. At high temperatures, it extrapolates to zero, whereas it levels out to a constant at low temperatures. For a wide range of temperatures in between, the data points display linear behaviour (without any evidence of logarithmic corrections), beginning at $\beta J=2.8$ down to $\beta J=9.0$. The exponent $\eta$ varies continuously from 0.323 at $\beta J=2.8$ to 0.092 at $\beta J=9.0$.

This is indeed close to the range expected from KT finite-size scaling (15), although there is an overestimate of the size of the KT phase when compared to the expected range of critical exponents, which is between $1 / 4$ and 1/9. As in the case of the Binder cumulant below, this is a simple consequence of the fact that the differ-

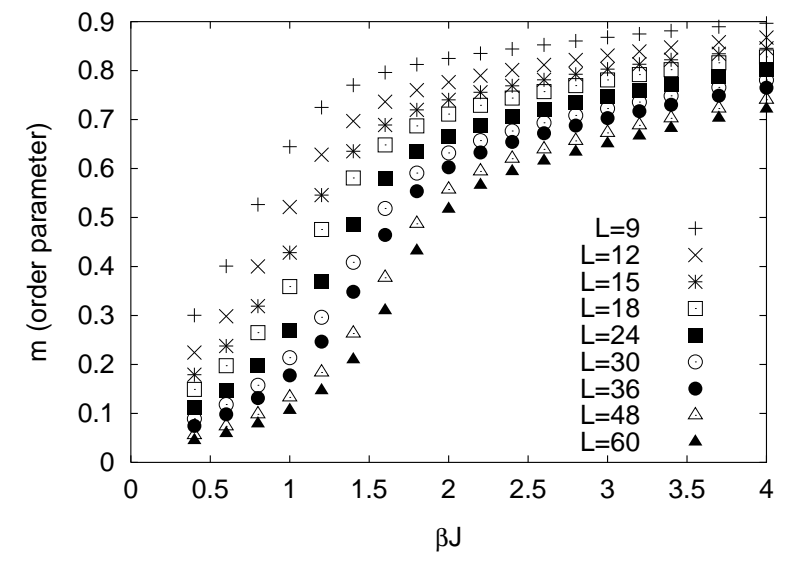

FIG. 2: The order parameter $m$ versus $\beta J$ for different system sizes.

ence between the ordered and the critical phase is indiscernible when the size of the system is much less than the correlation length. As the latter grows near the transition, increasingly large finite-size systems in the ordered phase appear to be critical.
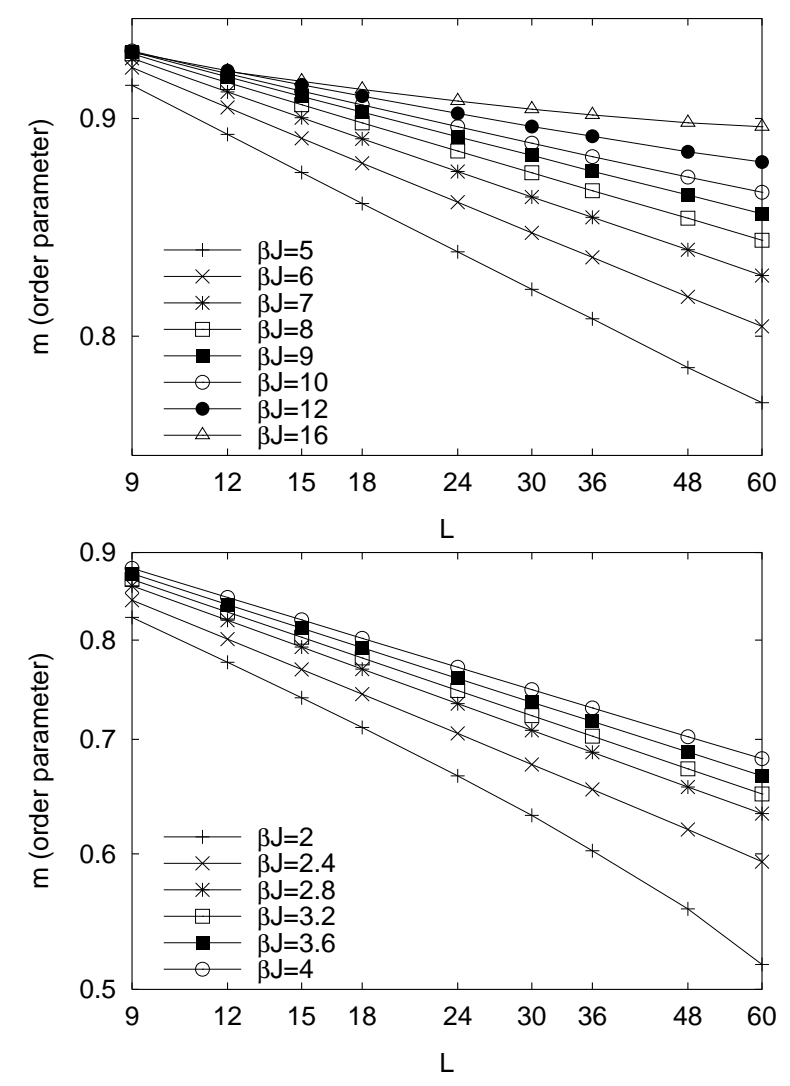

FIG. 3: The order parameter $m$ versus system size $L$ at various temperatures. The lines connect the points to guide the eye.

To check whether this critical region is indeed delin-

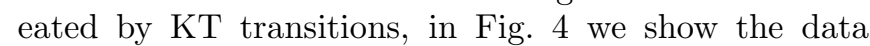
collapse (Eq. 17) for the upper transition. The data 
scales very well with the following set of parameters $\beta_{2} J=3.5, a=5.36, c=1.736$. Thus we can conclude that the system has a transition between the disordered phase and KT phase at $\beta_{2} J=3.5 \pm 0.2$. The critical exponent $\eta=0.263 \pm 0.015$ at the transition point. This value of the critical exponent $\eta$ is close to the theoretical prediction $1 / 4$, and the overestimate appears to be part of a systematic trend discussed below.

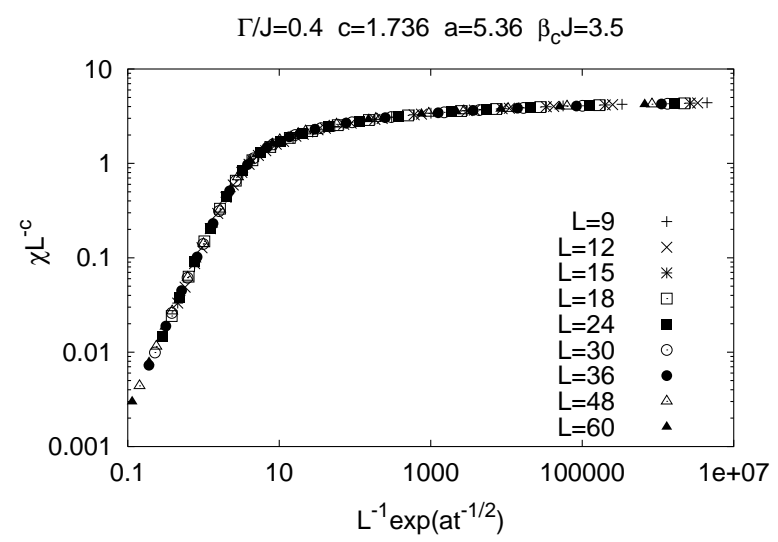

FIG. 4: Data collapse of the susceptibility for the upper transition.

In Fig. [5 we show the lower temperature scaled data (see Eq.16). The data scales quite well (but not perfectly, and over a narrower range than for the upper transition) with the following set of parameters: $\beta_{1} J=8.0, a=$ $1.2, c=0.105$. The error in determining the critical temperature is larger than in the high temperature case. We can conclude that the system has a transition between the KT phase and the ordered phase at $\beta_{1} J=8.0 \pm 1.0$. The critical exponent $\eta=0.105 \pm 0.02$ at the transition point. This value of the critical exponent $\eta$ is again close to the theoretical prediction $1 / 9$.

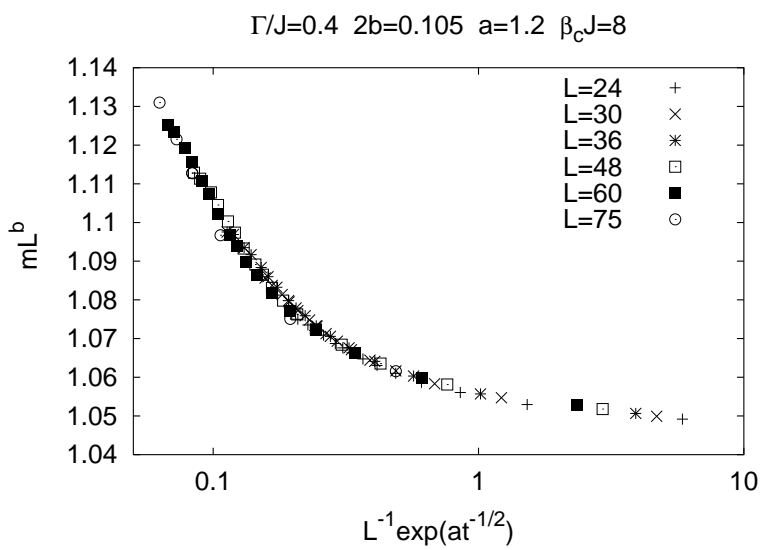

FIG. 5: Data collapse of the order parameter $m$ for the lower transition.

To determine the nature of the ordered phase, we consider the behaviour of $\cos (6 \theta)$ (Eq. 10). The plot of $\cos (6 \theta)$ as a function of the inverse temperature $\beta$ is shown in Fig. 6] $\cos (6 \theta)$ goes to -1 as the tempera- ture approaches zero. This implies the existence of the $(+0-)$-phase at low temperatures for $\Gamma / J=0.4$.

We can also determine the lower transition temperature $T_{1}$ from the criterion of $\eta=1 / 9$ at the lower transition point, which yields $\beta_{1} J=7.5 \pm 0.5$. At the transition temperature thus determined, one finds a crossing of $\cos (6 \theta)$ as a function of $\beta$ for different system sizes ${ }^{30}$

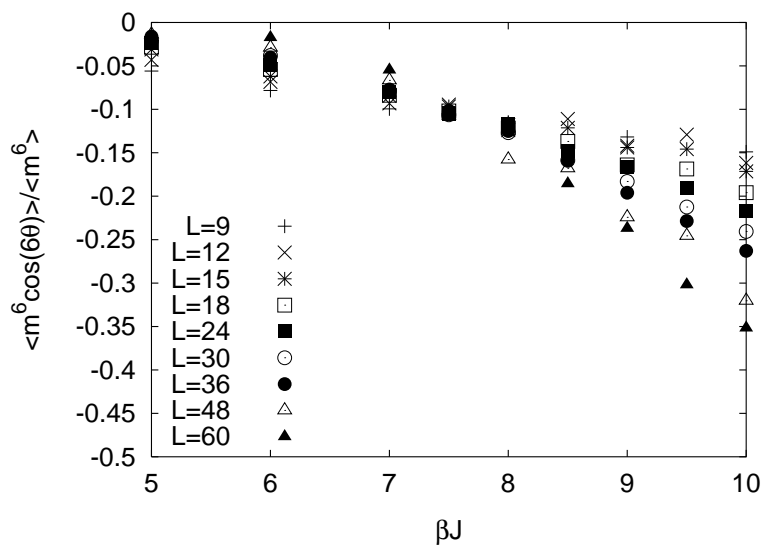

FIG. 6: The order parameter $\cos (6 \theta)$ versus $\beta J$ for different system sizes.

Next, we consider the flow diagram of the Binder cumulant, depicted in Fig. 7 In the high (low) temperature phase, its value approaches $0(2 / 3)$ as $L \rightarrow \infty$, whereas it levels off to a value $U^{*}$ in the KT phase which depends on the strength of the coupling. Challa and Landau ${ }^{28}$ proposed to use this levelling off as a diagnostic for the KT phase. We do indeed observe such a levelling over a wide range of temperature at $\Gamma / J=0.4 J$.

This diagnostic again systematically overestimates the extent of the critical phase. This is evidenced by the uppermost curves in Fig. 7 There is an inflection point at large system size where the value of $U_{L}$, having apparently levelled off, starts increasing again. As one approaches the transition from the ordered phase, this point of inflection wanders to increasingly larger system sizes and hence beyond the scope of the simulations.

\section{B. Other values of $\Gamma$}

We now describe what we have found for other values of $\Gamma$. At small $\Gamma / J \ll 1, T / J \ll 1, J$ drops out as an energy scale as the Hilbert space is restricted to the classical ground states, with excited states frozen out by an energy gap of $O(J)$. The only dimensionless parameter is then $\beta \Gamma$, so that the lines emanating from the origin of the phase diagram are straight. We estimate their slopes to be $T_{K T}^{l} / \Gamma=0.41 \pm 0.05$ for the lower transition, and for the upper: $T_{K T}^{u} / \Gamma=0.86 \pm 0.09$

The higher temperature data collapse does not always give the critical exponents $\eta$ at critical temperatures close to $1 / 4$, especially for large $\Gamma$. For example, we get the following upper set of critical temperatures 

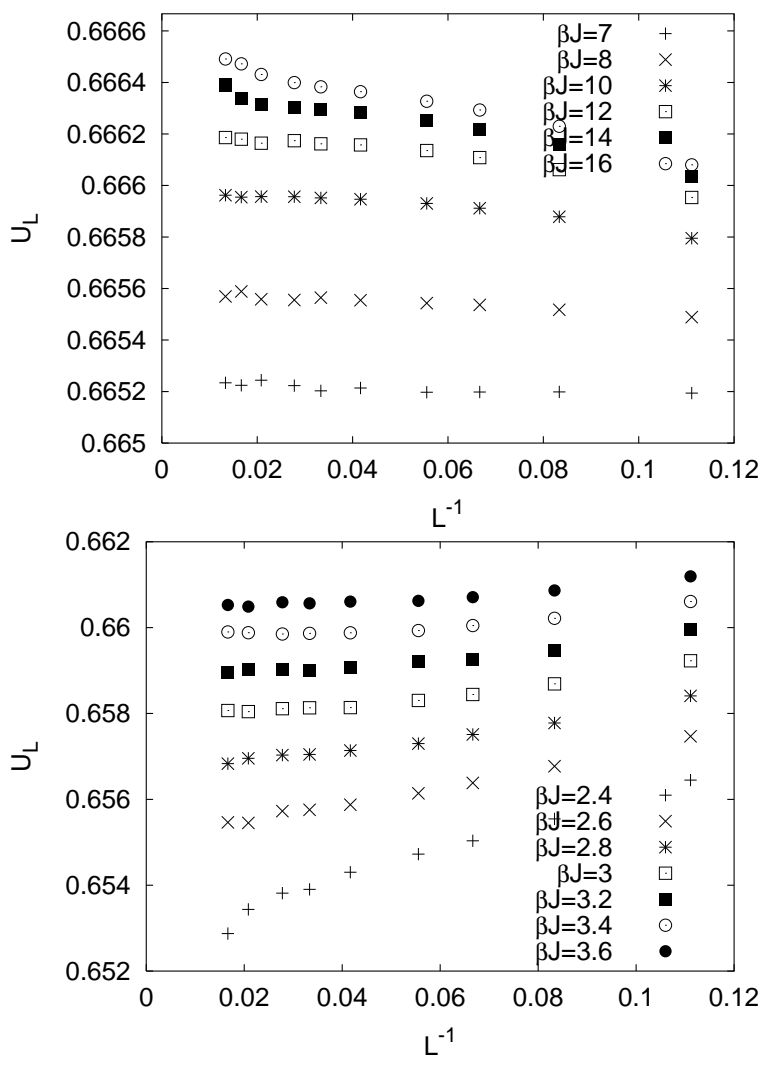

FIG. 7: The Binder cumulant $U_{L}$ versus system size $L$ at various temperatures. $\Gamma / J=0.4$. Note the different scales for the ordinate.

and critical exponents $\eta: \beta_{c} J=5.8(6), \eta=0.287(25)$ at $\Gamma / J=0.2, \beta_{c} J=3.5(2), \eta=0.263(15)$ at $\Gamma / J=0.4$, $\beta_{c} J=2.6(2), \eta=0.230(20)$ at $\Gamma / J=0.8$, and $\beta_{c} J=$ $3.0(3), \eta=0.203(25)$ at $\Gamma / J=1.2$. For $\Gamma / J=0.2$, the high-temperature data scales very well over a wide range of temperatures. Thus it is difficult to determine the precise value of the upper transition temperature at this $\Gamma$.

The systematic trend of decreasing $\eta$ with increasing $\Gamma$ is in accordance with the fact that $\eta$ at the zero temperature transition at large $\Gamma$ is different. For the $d=3$ $\mathrm{XY}$ universality class, $\eta$ is in fact close to 0,31 so that the increasing proximity of this fixpoint should be expected to show up in a correction of this kind. One can account for the systematic trend of increasing $\eta$ with decreasing $\Gamma$ in the same way. Indeed, the frustrated triangular Ising model has a critical point at $T=0$, where $\eta=1 / 2,32$

Probably related to this crossover is the fact that, as $\Gamma$ increases, the plots of the Binder cumulant as a function of system size fail to display the clear flattening visible in Fig. 7 for $\Gamma / J=0.4$ until a system size which is substantially larger; this is displayed for $\Gamma / J=0.8$ in Fig. 8

The lower transition at larger $\Gamma$ poses a problem for the alogrithm we use. Since we used linked lists to store the location of the domain walls in the temporal direction, the algorithm slows down considerably as $\Gamma$ increases. Together with the increasingly slowly decaying correla-
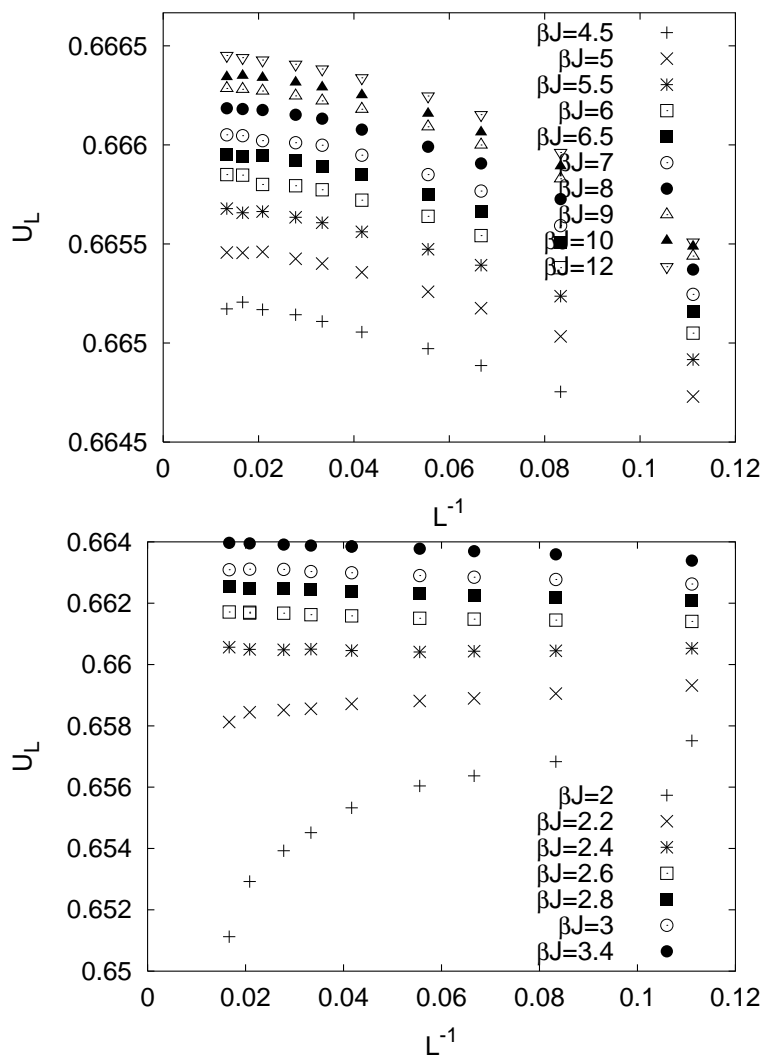

FIG. 8: The Binder cumulant $U_{L}$ versus system size $L$ at various temperatures. $\Gamma / J=0.8$. Note the different scales for the ordinate.

tions in the space direction as the temperature is lowered, this leads to a considerable increase in the correlation times of our algorithm. As a result, the data we were able to obtain were too noisy to permit a useful data collapse. We can, nonetheless, try to identify the location of the transition by tracking where $\eta$ crosses $1 / 9$ (Fig. (1)

We note that the classical six-state clock model, which has a phase diagram very similar to the one we find here, has been studied by many authors $\frac{28.33}{}$ As is the case for us, almost all the authors report that it is much more difficult to get data collapse at the lower KT transition than at the higher. Only in a recent paper, Tomita and Okabe,$\stackrel{34}{=}$ using a 'probability-changing cluster algorithm', have found the lower transition easily. Given the frustrated and higher dimensional nature of our spin model, such a cluster algorithm is not available to us.

The nature of the ordered phase at low $\Gamma$ is of the $(+0-)$ type. There is in principle the possibility of a change of sign of $v_{6}$ for entropic reasons as the couplings are varied .9 .19 As $\Gamma$ increases, it becomes increasingly hard to determine the sign of $\cos (6 \theta)$ for the system sizes available to us. The largest $\Gamma$ for which we can confidently state that the lower $\mathrm{KT}$ transition is into the $(+0-)$ phase is $\Gamma / J=1.2$. Although this difficulty may be in part due to a decrease in the strength of $v_{6}$, there is no supporting numerical evidence that the sign of $v_{6}$ ever changes. 


\section{The quantum critical point}

We now turn to the properties of the quantum critical point, i.e. to the zero temperature transition from the clock symmetry broken ordered phase to the paramagnet. We can determine its approximate location to be at $\Gamma_{c} / J=1.65 \pm 0.05$. For large $\Gamma$, the magnet is in a quantum paramagnetic phase with a size-independent susceptibility.

We have not directly attempted to determine the properties of this transition as extensive previous simulations on the classical stacked magnet exist. At the time, there were some suggestions that the observed critical exponents were in fact not those of a $d=3 X Y$ model 14 and perhaps altered due to the presence of another instability 12.13

The structure of our phase diagram lends support to the $d=3 X Y$ universality class scenario via an independent route. By inducing a dimensional crossover through switching on a finite temperature, we find that the highly nontrivial phase diagram is that predicted by the same Landau theory which gives the $d=3 X Y$ universality class. This diagnostic is perhaps more robust than a direct determination of the critical exponents, which can be influenced by corrections to scaling or the proximity of other instabilities.

The shape of the phase boundary near the quantum critical point follows from the knowledge of the critical exponents of the quantum phase transition 35 The boundaries of the KT phase near $\Gamma_{c}$ follow the trajectory $T_{K T} \propto\left|\Gamma-\Gamma_{c}\right|^{\nu z}$, where, for the present case, the dynamical critical exponent $z=1$ and $\nu$ is close to $2 / 3$

\section{CONCLUSION}

We have demonstrated that the common action of thermal and quantum fluctuations in the triangular lattice transverse field Ising model generate an interesting fluctuation-driven phase diagram including an extended critical phase bordered by a pair of KT transitions. We have employed several diagnostics for the presence of the KT phase and our results are consistent in considerable detail with what one would expect from an analysis based on a $d=2+1$ dimensional $X Y$ symmetric action with a six-fold clock anisotropy for a temporal dimension of tunable extent. This provides indirect support for the $X Y$ nature of the quantum critical point. The dynamical properties of the critical phase in close proximity to the quantum critical point are a subject worthy of a study in their own right.

The agreement between numerics and theory, however, is not complete. There are some deviations from the predicted critical exponents, which are probably due to a crossover to the zero temperature critical points. As we have used several diagnostics to determine the nature and location of phases and transitions, we are in a position to compare their relative reliability and find that, for the phase transitions in particular, considering the system size independence of the Binder cumulant and the power-law dependence of the magnetisation on system size systematically overestimate the extent of the critical phase.

In summary, the results we have obtained demonstrate once again that frustrated magnets provide a good starting point for finding unconventional phases and phase diagrams. In this particular case, by using a tunable combination of thermal and quantum fluctuations, we have managed to realise a standard model from statistical mechanics, the $X Y$ model with sixfold clock anisotropy 10 in dimensions $d=2$ and $d=3$, in terms of another one, namely the Ising model on the triangular lattice. Given other magnets in this class realise unusual order parameters,,$\stackrel{8}{=}$ this approach should provide more opportunities for studying exotic phase diagrams based on simple model spin systems.

\section{Acknowledgements}

We would like to thank D. Huse, A. Karlhede and especially S. Sondhi for many useful suggestions and discussions throughout the course of this work. RM is also grateful to P. Chandra and S. Sondhi for collaboration on closely related work. In addition, we thank A. Bunker, S. Fujiki, T. H. Hansson, J. Lidmar, and M. Oshikawa for useful discussions. This work was in part supported by the Ministère de la Recherche et des Nouvelles Technologies with an ACI grant.
1 For a non-technical introduction to frustrated magnets, see R. Moessner, Can. J. Phys. 79, 1283 (2001); reviews of exact diagonalisations and experiments, respectively, are C. Lhuillier, P. Sindzingre, and J.-B. Fouet, cond-mat/0009336 and P. Schiffer and A. P. Ramirez, Comments Cond. Mat. Phys. 18, 21 (1996).

2 J. Villain, R. Bidaux, J. P. Carton, and R. J. Conte, J. Phys. Paris 411263 (1980).

3 E. F. Shender, Sov. Phys. JETP 56, 178, (1982).

${ }^{4}$ C. L. Henley, Phys. Rev. Lett. 62, 2056 (1989).
5 see, e.g. K. Damle and S. Sachdev Phys. Rev. B 56, 8714 (1997) and S. Sachdev, cond-mat/9508080 for a pedagogical review.

6 J. Brooke, T.F. Rosenbaum, and G. Aeppli, Nature 413, 610 (2001); G. Aeppli, talk at conference on the Physics of Frustration, Santa Fe, 2002 (unpublished).

7 G. H. Wannier, Phys. Rev. 79, 357 (1950); R. M. F. Houtappel, Physica 16, 425 (1950).

${ }^{8}$ R. Moessner, S. L. Sondhi, and P. Chandra, Phys. Rev. Lett. 84, 4457 (2000), Phys. Rev. B 64, 144416 (2001); 
R. Moessner and S. L. Sondhi, Phys. Rev. B 63, 224401 (2001).

9 D. Blankschtein, M. Ma, A.N. Berker, G.S. Grest and C.M. Soukoulis, Phys. Rev. B 29, 5250 (1984).

10 J. V. Jose, L. P. Kadanoff, S. Kirkpatrick and D. R. Nelson, Phys. Rev. B 16, 1217 (1977).

11 P. Chandra, P. Coleman and L. B. Ioffe, Phys. Rev. B 49, 12897 (1994).

12 O. Heinonen and R. G. Petschek, Phys. Rev. B 40, 9052 (1989).

13 R. R. Netz and A. N. Berker Phys. Rev. Lett. 66, 377 (1991).

14 Alex Bunker, B. D. Gaulin, and C. Kallin, Phys. Rev. B 48, 15861 (1993).

15 M. L. Plumer and A. Mailhot, Physica A222, 437 (1995).

${ }^{16}$ For a review, see M. F. Collins and O. A. Petrenko, Can. J. Phys. 75, 605 (1997).

17 M. Suzuki, Prog. Theor. Phys. 46, 1337, (1971); ibid 56, 2454 (1976).

18 H. F. Trotter, Proc. Am. Math. Soc. 10, 545 (1959).

19 N. Todoroki, Y. Ueno, and S. Miyashita, Phys. Rev. B 66, 214405 (2002).

20 D. J. Amit, Field Theory, the Remormalization group and Critical Phenomena, World Scientific (1984).

21 B. B. Beard and U.-J. Wiese, Phys. Rev. Lett. 77, 5130 (1996).

22 N. V. Prokof'ev, B. V. Svistunov, and I. S. Tupitsyn,
Pis'ma Zh. Eks. Teor. Fiz. 64, 853 (1996) [JETP Lett. 64, 911 (1996)]; Zh. Eks. Teor. Fiz. 114, 570 (1998) [JETP 87, 311 (1998)].

${ }^{23}$ H. Rieger and N. Kawashima, Eur. Phys. J. B 9, 233, (1999).

24 R. H. Swendsen and J.-S. Wang, Phys. Rev. Lett. 58, 86 (1987).

25 U. Wolff, Phys. Rev. Lett. 62, 361 (1989).

26 For a pedagogical introduction, see W. Krauth, cond-mat/9612186

27 K. Binder, Z. Phys. B 43, 119 (1981).

28 M. S. S. Challa and D. P. L. Landau Phys. Rev. B 33, 437 (1986).

29 J. Kosterlitz, J. Phys. C 7, 1046 (1974).

30 Indeed, M. Oshikawa (Phys. Rev. B 61, 3430 (2000)) notes that $\cos (6 \theta)$ has a scaling dimension of zero for the six-state clock model.

31 R. Guida and J. Zinn-Justin, J. Phys. A 31, 8103 (1998).

32 J. Stephenson, J. Math. Phys. 11, 413 (1970).

33 S. Fujiki, K. Shuton, S. Inawashiro, Y. Abe, and S. Katsura, J. Phys. Soc. Jpn. 55, 3326 (1986); A. Yamagata and I. Ono, J. Phys. A 24, 265 (1991); S. Fujiki and T. Horiguchi, J. Phys. Soc. Jpn. 64, 1293 (1995).

34 Y. Tomita and Y. Okabe, Phys. Rev. B 65, 184405 (2002).

${ }^{35}$ For reviews, see S. L. Sondhi, S. M. Girvin, J. P. Carini and D. Shahar, Rev. Mod. Phys. 69, 315 (1997), or Ref. 5. 